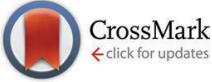

Cite this: New J. Chem., 2016, 40,4414

Received (in Montpellier, France) 11th January 2016,

Accepted 29th March 2016

DOI: $10.1039 / c 5 n j 03668 b$

www.rsc.org/njc

\title{
Influence of the pore architecture on the selective conversion of ethene to propene and butenes over medium pore zeolites
}

\author{
S. Follmann and S. Ernst*
}

\begin{abstract}
This contribution focuses on the conversion of ethene to propene and butenes over medium pore zeolites with different pore architectures and similar $n_{\mathrm{Si}} / n_{\mathrm{Al}}$-ratios. The aim of this systematic study was to explore the factors which govern the yield/selectivity of propene/butenes from ethene and to optimize the catalytic properties for this reaction. All used zeolites were prepared by hydrothermal synthesis and characterized by various techniques. Their catalytic properties (with respect to increased propene yield/selectivity) were explored in a fixed-bed flow-type reactor operating under atmospheric pressure. One major result of these investigations is that medium pore zeolites with a one-dimensional channel system offer catalytic properties superior to the well-known zeolite ZSM-5 with its threedimensional pore system. Selectivities to propene of $43 \%$ and to butenes of $24 \%$ could be achieved over zeolite HZSM-23 used as the catalyst at medium conversions. This demonstrates the effective suppression of by-product formation over a wide range of reaction conditions due to shape selectivity effects in the tubular pore structure of HZSM-23.
\end{abstract}

\section{Introduction}

The world-wide consumption of light olefins in the chemical industry is steadily increasing. It is expected that especially the high demand for polymers will lead to a continuing strong growth rate of about $4 \%$ for ethene and $5 \%$ for propene. In the last few years, the market for propene has grown faster than the one for ethene, which resulted in the so-called propene gap. ${ }^{1}$ So far, most of the light olefins required by the petrochemical industry were obtained via steam cracking of different feedstocks. Depending on the location of the steam cracker and feedstock availability, naphtha or ethane are predominantly used as feedstocks, beside gas oil and NGL (natural gas liquids). The shale gas boom in certain regions of the world promotes an increasing use of shale gas-derived ethane as a raw material due to low feedstock prices. This trend is affecting the availability of propene, butenes and aromatics in a negative manner. With respect to the product distribution, a significantly higher yield of ethene is obtained by steam cracking of ethane as compared to other feedstocks. Only small amounts of propene, butenes and pyrolysis gasoline are produced in comparison to naphtha steam cracking. ${ }^{2,3}$ It is obvious that one of the biggest upcoming challenges in the petrochemical industry is the future supply of propene and butenes.

Technische Universitat Kaiserslautern, Department of Chemistry,

Erwin-Schroedinger Str. 54, Kaiserslautern, Germany.

E-mail: ernst@chemie.uni-kl.de
New strategies, the so-called on-purpose processes, were developed to meet the increasing demand for propene independently from its production via steam cracking. Among them are fluid catalytic cracking (FCC), ${ }^{3}$ the metathesis of ethene with butenes, ${ }^{4}$ propane dehydrogenation ${ }^{5}$ and the methanol-topropylene (MTP) or methanol-to-olefin (MTO) route. ${ }^{6}$ All these processes are already available on an industrial scale. A novel on-purpose technology is the direct conversion of ethene to propene and butenes (ETP-reaction). The advantage of the ETPreaction is a high feedstock flexibility, because ethene can not only be obtained from crude oil as feedstock (after steam cracking) but also from other sources like shale gas and natural gas (after dehydrogenation of ethane), as well as coal (via syngas) and biomass (via bioethanol). ${ }^{7}$

The development of more efficient zeolite catalysts in the ETP-reaction requires more detailed studies of the factors influencing their activity and selectivity. Various catalysts have been described in the literature, including supported metal oxide catalysts, ${ }^{8}$ mesoporous M41S materials ${ }^{9}$ and microporous zeolites. ${ }^{10-13}$ Particularly zeolites are promising catalysts due to their versatile properties like tunable pore structure, shape selectivity, acidity and thermal/hydrothermal stability.

The focus of this systematic study was to explore the factors which govern the yield/selectivity of propene/butenes from ethene and to optimize the catalytic properties for this reaction. For this purpose, zeolites with different pore sizes/architectures (viz. Beta, ZSM-5, ZSM-11, ZSM-22, ZSM-23, EU-1) were synthesized, 
transformed into their acid forms and explored for their catalytic properties in a fixed-bed flow-type apparatus.

\section{Experimental}

\section{Synthetic procedures}

The zeolites used in the present study were synthesized using hydrothermal methods. In order to crystallize zeolites ZSM-5 and ZSM-11, tetra- $n$-propylammonium bromide and tetra- $n$-butylphosphonium bromide were used as structure directing agents. ${ }^{14}$ Zeolites ZSM-22 and ZSM-23 were synthesized using procedures described by Ernst et al. using 1,6-diaminohexane and pyridine, respectively, as template molecules. ${ }^{15,16}$ Zeolite EU-1 was prepared with hexamethonium bromide and zeolite Beta was synthesized using tetraethylammonium hydroxide as an organic template according to the published procedures. ${ }^{17}$

The as-synthesized materials were washed with distilled water and dried at $120{ }^{\circ} \mathrm{C}$ over night. The dry materials were heated to $560{ }^{\circ} \mathrm{C}$ with a rate of $1{ }^{\circ} \mathrm{C} \mathrm{min}{ }^{-1}$ and kept at the final temperature for $24 \mathrm{~h}$. The acid zeolites were prepared via ionexchange using a 0.25 molar ammonium nitrate solution for three hours at $80{ }^{\circ} \mathrm{C}$. This procedure was repeated twice. After a further calcination step at $560{ }^{\circ} \mathrm{C}$, the catalysts were pelletized, crushed and sieved and the particle size fraction between 250 and $355 \mu \mathrm{m}$ was used for the catalytic experiments.

\section{Catalytic experiments}

The conversion of ethene was carried out in a fixed-bed flowtype reactor under atmospheric pressure. For each catalytic experiment, $500 \mathrm{mg}$ of the fresh catalyst was transferred into a quartz glass reactor and dried in a vertical furnace at $600{ }^{\circ} \mathrm{C}$ for $0.5 \mathrm{~h}$ before cooling down to the desired reaction temperature. Ethene was diluted with high purity nitrogen $(>99,99 \%)$ as a carrier gas and fed into the reactor via a mass flow controller. Unless otherwise stated, the partial pressure of ethene typically

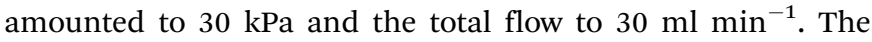
time-on-stream behavior of all catalysts was followed over at least $24 \mathrm{~h}$. Detailed product distributions are given for $0.5 \mathrm{~h}$ time-on-stream. The product composition was analyzed via gas chromatography (Agilent 6890) equipped with a flame ionization detector (FID). The separation of hydrocarbons was carried out using an HP-PLOT M capillary column (length $50 \mathrm{~m}$, inner diameter $0.53 \mathrm{~mm}$ ). All tubes and valves following the reactor outlet were heated to $180{ }^{\circ} \mathrm{C}$, to prevent condensation of heavier hydrocarbon products.

\section{Characterization}

Powder X-ray diffraction (XRD) pattern were collected using a Siemens D5005 instrument using $\mathrm{CuK}_{\alpha}(40 \mathrm{kV}, 30 \mathrm{~mA})$ radiation to control crystallinity and phase purity of the synthesized zeolites. Nitrogen physisorption isotherms at $-196{ }^{\circ} \mathrm{C}$ were obtained using a Quantachrome Autosorb-1 apparatus. The determination of the specific surface areas was done according to the BET procedure. ${ }^{18}$ For this purpose, samples were degassed at $200{ }^{\circ} \mathrm{C}$ for $24 \mathrm{~h}$ under vacuum $\left(10^{-1} \mathrm{~Pa}\right)$. Thermal analyses (TGA) were carried out using a Setaram Setsys 16/18 instrument. In a typical procedure, approximately $40 \mathrm{mg}$ of the material was heated in air from $40{ }^{\circ} \mathrm{C}$ to $900{ }^{\circ} \mathrm{C}$ with a heating rate of $10{ }^{\circ} \mathrm{C} \mathrm{min}{ }^{-1}$. An observed weight loss in the range of $400-750{ }^{\circ} \mathrm{C}$ was assumed to result from coke burn-off. Atomic absorption spectroscopy (AAS) was performed using a Perkin Elmer AAnalyst 400 instrument to determine the $n_{\mathrm{Si}} / n_{\mathrm{Al}}$-ratios of the synthesized zeolites. Scanning electron micrographs (SEM) were collected using a Jeol microscope (JSM-6490LA) to characterize the size and morphology of the materials used in this study. The samples were sputtered with a thin layer of gold $(15 \mathrm{~nm})$ to increase contrast and thereby also the possible magnification.

\section{Results and discussion}

\section{Catalyst characterization}

Powder X-ray diffraction pattern of the as-synthesized zeolites are shown in Fig. 1. All materials exhibit a good crystallinity which is indicated by high signal intensities. No indications of phase impurities or amorphous materials could be observed. ${ }^{19}$

The specific pore volumes and the specific surface areas of the acid catalysts were determined using the BET-method. The results are summarized in Table 1. The BET-surface areas and the total pore volumes are in the expected range for the investigated materials.

Scanning electron microscopy was used to determine the morphology and size of the zeolite crystallites. The results are shown in Fig. 2.

Zeolite Beta consists of small crystallites with a mean size below $1 \mu \mathrm{m}$. Zeolite ZSM-5 shows the typical morphology, viz.

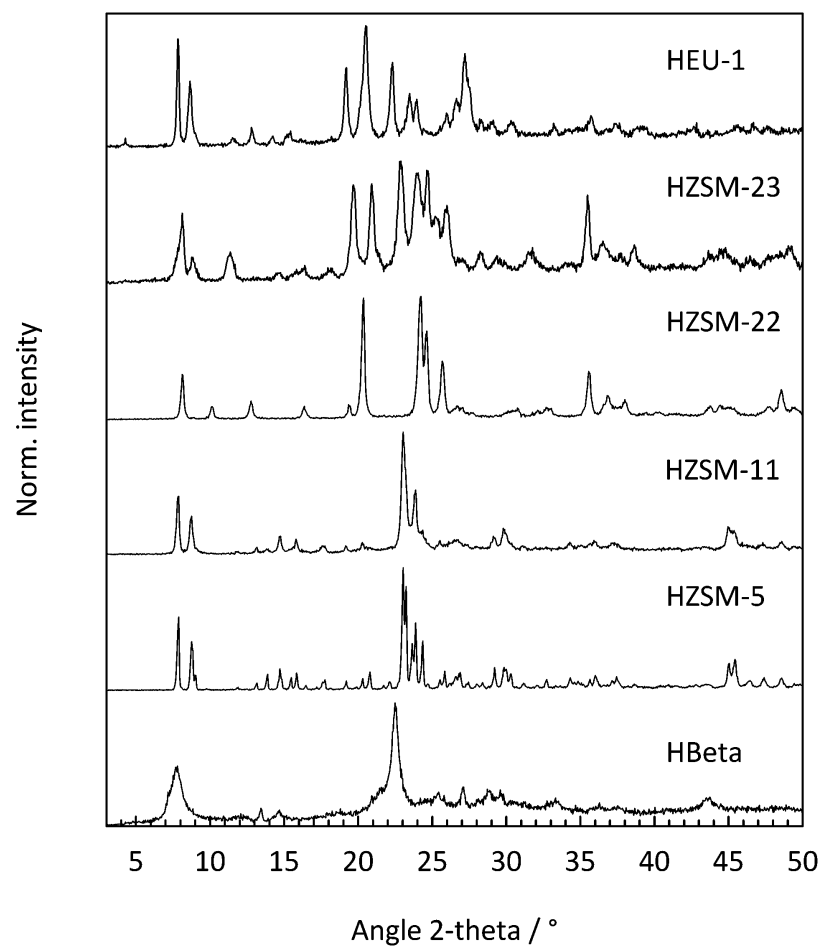

Fig. 1 Powder X-ray diffraction pattern (XRD) of the synthesized zeolites. 
Table 1 Textural properties and chemical compositions of the synthesized zeolites used in this study

\begin{tabular}{llll}
\hline Material & $S_{\mathrm{BET}} / \mathrm{m}^{2} \mathrm{~g}^{-1}$ & $V_{\text {por. }} / \mathrm{cm}^{3} \mathrm{~g}^{-1}$ & $n_{\mathrm{Si}} / n_{\mathrm{Al}}$-ratio \\
\hline HBeta & 880 & 0.38 & 27 \\
HZSM-5 & 470 & 0.21 & 37 \\
HZSM-11 & 500 & 0.24 & 35 \\
HZSM-22 & 300 & 0.15 & 35 \\
HZSM-23 & 340 & 0.19 & 34 \\
HEU-1 & 470 & 0.19 & 33
\end{tabular}

hexagonal prisms with crystallite sizes of $c a .5 \mu \mathrm{m}$ in length and a thickness of $c a .1 .5 \mu \mathrm{m}$. The crystallites of zeolite ZSM-11 are spherical-like with mean diameters of $c a$. $1 \mu \mathrm{m}$. For zeolite ZSM-22 rod-like crystallites can be observed with lengths up to $3 \mu \mathrm{m}$. Zeolite ZSM-23 as prepared in the present study consists of radial fin shaped rods, which agglomerates to larger particles with a size of about $1 \mu \mathrm{m}$. EU-1 consists of small elliptical particles with a length of around $3 \mu \mathrm{m}$ and a diameter of approximately $2-3 \mu \mathrm{m}$.

\section{Catalytic performance of 10-membered ring zeolites}

Important criteria for the selection of a suitable catalyst for a given reaction are its activity, selectivity and the time-on-stream behavior. The focus is here on a structure/property relationship of different well-known zeolites for the ETP-reaction. The following catalytic experiments were carried out under similar reaction conditions and with zeolite samples of comparable $n_{\mathrm{Si}} / n_{\mathrm{Al}}$-ratios. In the first step we investigated the influence of the pore architecture of the zeolite catalysts on the time-onstream behavior at $550{ }^{\circ} \mathrm{C}$ (Fig. 3).

The time-on-stream stabilities decrease in the following direction: HZSM-11 > HZSM-5 > HZSM-23 > HZSM-22 > HEU-1 > HBeta. The results clearly indicate a continuous decrease of activity with extended time-on-stream which is a

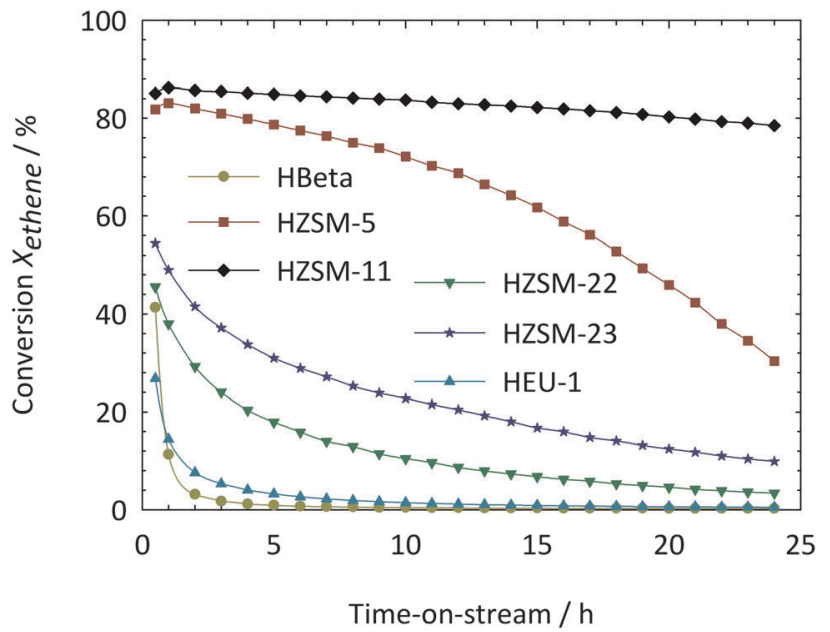

Fig. 3 Time-on-stream behavior of acid zeolites with similar $n_{\mathrm{Si}} / n_{\mathrm{Al}}$-ratios. Reaction conditions: $T=550{ }^{\circ} \mathrm{C}, p_{\text {(ethene) }}=30 \mathrm{kPa}, F_{\text {total }}=30 \mathrm{ml} \mathrm{min}{ }^{-1}$, $W_{\text {cat. }}=500 \mathrm{mg}$.

result of the high coking tendency of ethene under the applied reaction conditions. The amount of coke which was formed after a continuous run of $24 \mathrm{~h}$ was investigated by thermal analyses (Table 2).

It can be seen that the coke content after $24 \mathrm{~h}$ time-on-stream is considerably higher for zeolite Beta with its three-dimensionally interconnected system of 12-membered ring pores as compared to the 10-membered ring zeolites. The coke contents of onedimensional systems are slightly lower than those of the threedimensional channel systems: over zeolites with one-dimensional channel systems, small amounts of coke deposited at the pore entrances or in the pores can lead to a complete blockage of the channels and the intrazeolitic active sites, hence the fast deactivation of the one-dimensional systems which can be seen
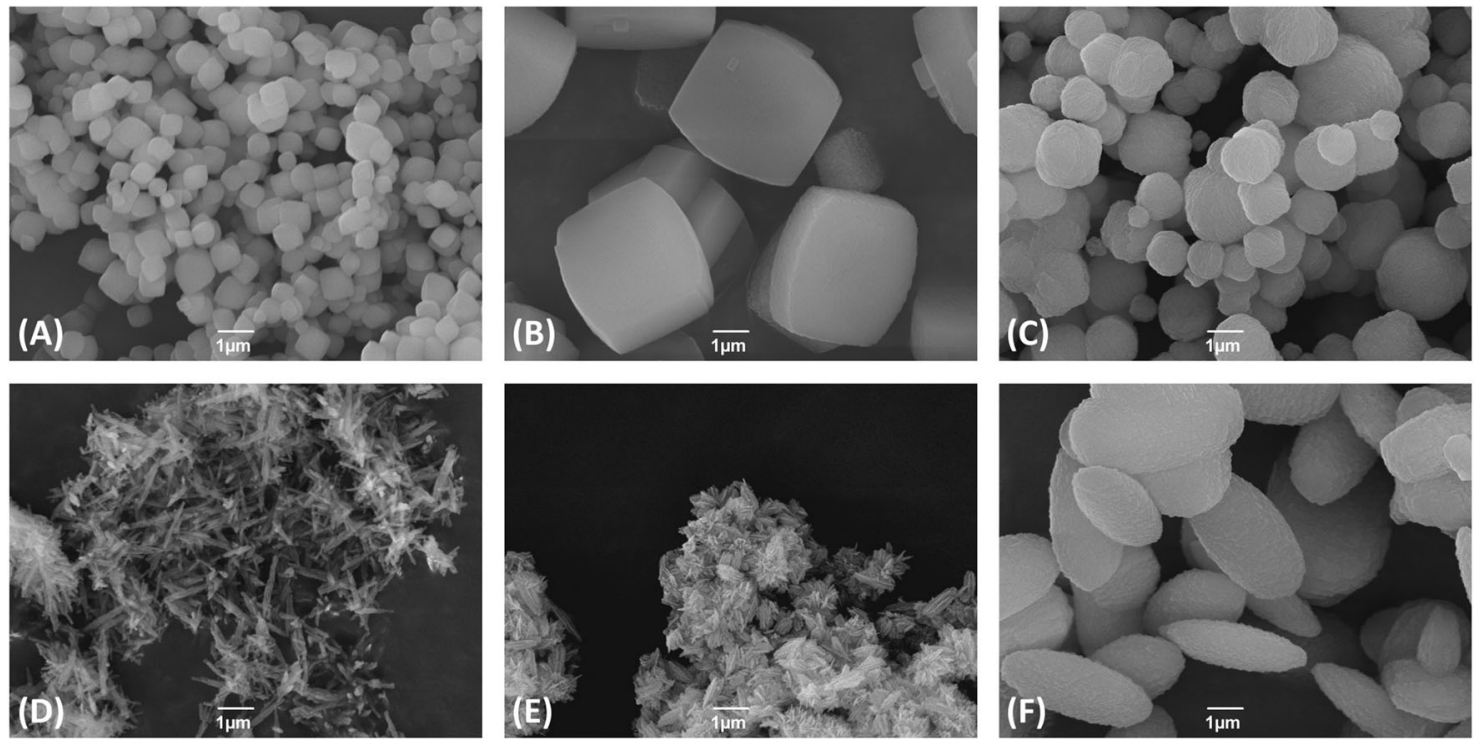

Fig. 2 Scanning electron micrographs (SEM) of the synthesized zeolites with a magnification of $10000 \times$ : (A) Beta, (B) ZSM-5, (C) ZSM-11, (D) ZSM-22, (E) ZSM-23, (F) EU-1. 
Table 2 Characterization of spent catalysts after $24 \mathrm{~h}$ time-on-stream

\begin{tabular}{lclc}
\hline Catalyst & $S_{\text {BET }} / \mathrm{m}^{2} \mathrm{~g}^{-1}$ & $V_{\text {por. }} / \mathrm{cm}^{3} \mathrm{~g}^{-1}$ & Coke/wt\% \\
\hline HBeta & 20 & 0.017 & 25.5 \\
HZSM-5 & 190 & 0.084 & 8.9 \\
HZSM-11 & 340 & 0.153 & 7.6 \\
HZSM-22 & 60 & 0.084 & 6.2 \\
HZSM-23 & 150 & 0.146 & 5.7 \\
HEU-1 & 20 & 0.013 & 5.7 \\
\end{tabular}

from Fig. 3. Particularly pronounced is the deactivation on the 12-membered ring pore architecture of zeolite Beta. The activity decay from initially $41 \%$ after $0.5 \mathrm{~h}$ is almost complete after $3 \mathrm{~h}$ of time-on-stream. The large void spaces in the pore structure lead to a strong coking activity which causes high coke loadings of $25.5 \%$ after $24 \mathrm{~h}$. The pore system is totally blocked by coke which is evident by $\mathrm{N}_{2}$-physisorption measurements of the spent catalyst (Table 2). The large pores of zeolite Beta offer enough space for the bulky transition states of the hydrogen transfer, aromatization and alkylation reactions of the light olefins. These secondary reactions promote the formation of coke either inside the pore system or on the external surface of the crystallites. The smaller pore openings of the threedimensional 10-membered ring pore structures of zeolites ZSM-5 and ZSM-11 reduce the amount of secondary reactions, whereby the activity and stability increase and the coke loadings decrease significantly. The initial ethene conversions over ZSM-5 and ZSM-11 amounted to $c a$. $82 \%$ and $c a$. 85\%, respectively, with final coke loadings of $8.9 \%$ and $7.6 \%$. The deactivation of both zeolites is very different despite very similar structures and coke loadings. This experimental observation is tentatively explained by the differences in the crystallite size of zeolites ZSM-5 and ZSM-11 as revealed by scanning electron microscopy. The corresponding one-dimensional 10-membered ring zeolites with a tubular pore system exhibit a stronger influence of coke deposition on activity and stability. This is particularly evident in the case of zeolites ZSM-22 and ZSM-23 with coke contents of $c a .6 .2 \%$ and $c a .5 .7 \%$, respectively and initial conversions of the time-of-stream behavior of $c a$. $46 \%$ and $c a .54 \%$, respectively. The deposition of coke has a stronger impact on conversion/selectivities over tubular pore structures (as compared to three-dimensional systems) by pore blocking effects leading to a faster catalyst deactivation. In addition, diffusion problems of reactants need to be considered which may have a pronounced influence on the catalytic performance of tubular pore structures. Although Zeolite HEU-1 also belongs to the family of one-dimensional 10-membered ring zeolites, clear differences were observed in activity and stability for ethene conversion. The activity of zeolite HEU-1 after $0.5 \mathrm{~h}$ of time-on-stream was estimated to be $27 \%$ with a final coke loading of $5.7 \%$ after $24 \mathrm{~h}$. The low activity and the very fast deactivation of zeolite HEU-1 compared to the other onedimensional pore structures can be tentatively attributed to the tubular pore system with large 12-membered ring side pockets which make this zeolite prone to coke formation with concomitant pore blockage.
Table 3 Influence of the pore architecture on the conversion and selectivities in the ETP-reaction

\begin{tabular}{|c|c|c|c|c|c|c|}
\hline \multirow[b]{2}{*}{$\begin{array}{l}\text { Catalyst }^{a} \\
\left(n_{\mathrm{Si}} / n_{\mathrm{Al}}-\text { ratio }\right)\end{array}$} & \multirow[b]{2}{*}{$\begin{array}{l}\text { Conversion } \\
X_{\text {ethene }} / \%\end{array}$} & \multicolumn{5}{|c|}{ Selectivity $/ \%$} \\
\hline & & Propene & Butenes & $\begin{array}{l}\mathrm{C}_{1}-\mathrm{C}_{4} \\
\text { alkanes }\end{array}$ & $\mathrm{C}_{5+}{ }^{b}$ & Aromatics \\
\hline HBeta (27) & 41.3 & 14.1 & 5.1 & 69.1 & 3.8 & 7.9 \\
\hline HZSM-5 (37) & 81.8 & 22.9 & 10.2 & 31.4 & 6.3 & 29.2 \\
\hline HZSM-11 (35) & 85.0 & 19.1 & 8.7 & 38.2 & 4.9 & 29.1 \\
\hline HZSM-22 (35) & 45.5 & 41.8 & 28.3 & 6.3 & 20.5 & 3.1 \\
\hline HZSM-23 (34) & 54.4 & 43.4 & 24.4 & 8.9 & 18.4 & 4.9 \\
\hline HEU-1 (33) & 26.7 & 45.4 & 21.1 & 13.2 & 14.7 & 5.6 \\
\hline
\end{tabular}

The observed fast deactivation of zeolite catalysts is a wellknown phenomenon in acid-catalyzed hydrocarbon conversions due to the formation of coke as an almost always inevitable side reaction. In principle, therefore, no steady- or stationary-state for ethene conversion can be observed. However, in order to compare the catalytic behaviour of the catalysts, we selected the product compositions which were obtained after $0.5 \mathrm{~h}$ time-on-stream. The conversion of ethene to propene and butenes produces a variety of reaction products. Depending on the formation mechanism and the carbon number of these products they were grouped in 5 fractions. These include propene and butenes as main reaction products as well as saturated $\mathrm{C}_{1}-\mathrm{C}_{4}$ alkanes, aromatics and aliphatic hydrocarbons with five and more carbon atoms $\left(\mathrm{C}_{5+}\right)$ as by-products. All tested zeolites possess high selectivities for the formation of propene and butenes under the applied reaction conditions (Table 3 ).

Among the zeolites studied in this work, the one-dimensional 10 -membered ring pore structure showed the highest total selectivities to propene and butenes in the range of $66 \%$ to $70 \%$. The three-dimensional 10-membered ring zeolites showed comparatively lower overall selectivities to these light olefins with approximately $28 \%$ to $33 \%$. The lowest selectivity to propene and butenes with $19 \%$ was observed with the very open pore structure of zeolite Beta. These results clearly indicate the key role of spatial constraints in the intracrystalline voids for the selective formation of propene and butenes. This is also reflected in the by-product formation, especially the formation of aromatics and saturated $\mathrm{C}_{1}-\mathrm{C}_{4}$ alkanes which are clearly reduced in one-dimensional systems. Increasing the dimensionality and the size of the pore opening results in the increase in selectivities for the formation of saturated $\mathrm{C}_{1}-\mathrm{C}_{4}$ alkanes (and aromatics). The reason for this behavior is probably related to an increased contribution of hydrogen transfer reactions with increasing space available around the catalytic sites.

\section{Influence of the temperature}

For further studies on the influence of the reaction temperature in the ETP-reaction, zeolites HZSM-5 and HZSM-23 were selected as potential catalysts. The determined product distributions with these two catalysts are shown for a temperature range of $300-600{ }^{\circ} \mathrm{C}$ in Fig. 4. 

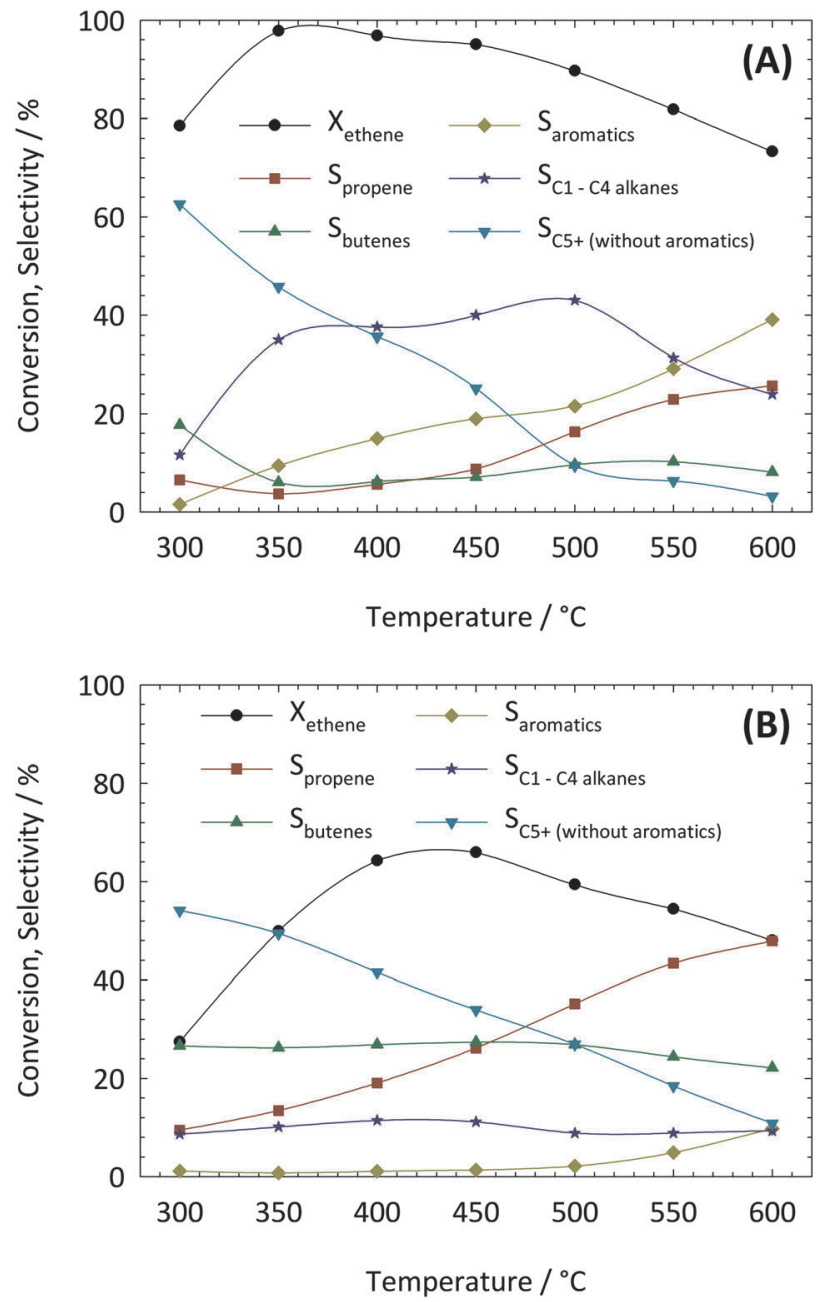

Fig. 4 Influence of the reaction temperature on catalytic performance of zeolites HZSM-5 (A) and HZSM-23 (B). Reaction conditions: $p_{\text {(ethene) }}=$ $30 \mathrm{kPa}, F_{\text {total }}=30 \mathrm{ml} \mathrm{min}{ }^{-1}, W_{\text {cat. }}=500 \mathrm{mg}, \mathrm{TOS}=0.5 \mathrm{~h}$.

The ETP-reaction consists of two elementary steps: the exothermic oligomerization of ethene to longer hydrocarbon chains and the endothermic cracking of higher hydrocarbons to short-chain olefins. In total, an exothermic reaction occurs, which means that thermodynamic equilibrium conversion decreases with increasing reaction temperature. This behavior is indeed observed with ZSM-5 and ZSM-23 (cf. Fig. 4). The differences in the conversion levels especially at lower reaction temperatures could be caused by mass transport limitations within the one-dimensional pore structure of zeolite ZSM-23.

The strong influence of the reaction temperature on the two competing reaction mechanisms (viz. mono- vs. bimolecular transition states) has a crucial impact on the product distribution. For both zeolites increasing selectivities to propene were observed with increasing reaction temperature while the selectivities to butenes showed no appreciable changes over the whole temperature range. Moreover, considerable amounts of aliphatic hydrocarbons $\mathrm{C}_{5+}$ (without aromatics) are formed at lower reaction temperatures, which then decrease significantly with increasing temperature. In the case of zeolite ZSM-23, no significant changes in selectivity to $\mathrm{C}_{1}-\mathrm{C}_{4}$ alkanes can be observed. The formation of aromatic products from ethene clearly starts at a temperature above $500{ }^{\circ} \mathrm{C}$. Zeolite ZSM- 5 , on the other hand, produces large amounts of saturated $\mathrm{C}_{1}-\mathrm{C}_{4}$ alkanes and aromatics over the whole temperature range.

\section{Influence of the partial pressure}

The influence of the partial pressure of ethene on the catalytic activities and selectivities was studied in the range from 10 to $100 \mathrm{kPa}$. As shown in Fig. 5, conversion increases with increasing ethene partial pressure of zeolites ZSM-5 and ZSM-23 up to $c a .30 \mathrm{kPa}$. Only a slight further increase in conversion is observed by increasing the ethene partial pressure to $100 \mathrm{kPa}$. In both "regimes", the selectivities to propene increase with decreasing $p_{\text {ethene. }}$. On the other hand, with increasing partial pressure of ethene the selectivities to $\mathrm{C}_{5^{+}}$hydrocarbons increase. This is most probably a result of co-oligomerization between ethene and propene, which becomes more pronounced at higher molar fractions in the feed gas. The selectivities to saturated $\mathrm{C}_{1}-\mathrm{C}_{4}$ alkanes and aromatics show only minor changes over the
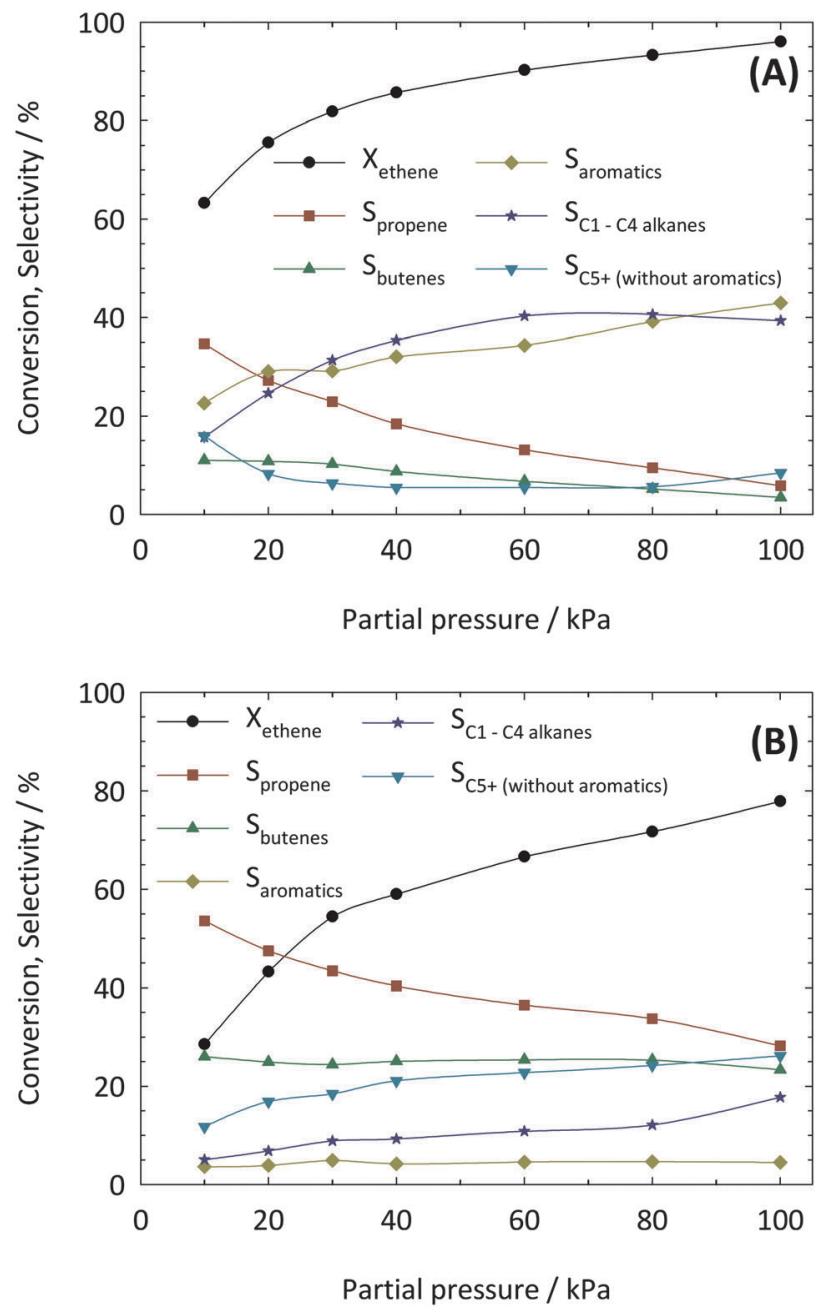

Fig. 5 Influence of the ethene partial pressure on conversion and selectivities over zeolites HZSM-5 (A) and HZSM-23 (B). Reaction conditions:

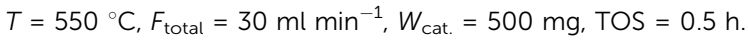


investigated range of ethane partial pressures. Presumably, this is an effect of unspecific reactions on the outer surface of the crystallites where no shape selectivity effects occur.

In contrast to zeolite ZSM-23, ZSM-5 showed clear differences in the by-product formation. Neither an influence of ethene partial pressure on the selectivity to $\mathrm{C}_{5+}$ hydrocarbons nor on those of saturated $\mathrm{C}_{1}-\mathrm{C}_{4}$ alkanes and aromatics was observed. Considering the selectivities to the saturated hydrocarbons it is clear that the involved cracking mechanisms depend strongly on the mole fraction of ethene in the gas feed. In this context, monomolecular cracking is favored at low partial pressures of ethene whereas bimolecular cracking and thus hydrogen transfer reactions are promoted at high partial pressures of ethene. Interestingly, a relationship between the formation of aromatics and propene can be observed (Fig. 5). The results suggest that aromatics are predominantly formed by secondary reactions of propene.

\section{Conclusions}

The results of the present study demonstrate the influence of the pore architecture of selected different zeolite structures on the catalytic conversion of ethene to propene and butenes. One major result of this study is that spatial constraints of the pore structure have a significant influence on the formation of propene and butenes. Here, the selectivities to propene and butenes decrease in the direction: HZSM-22 > HZSM-23 >

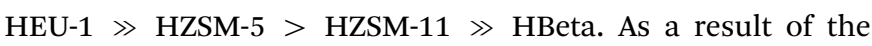
decreasing spatial constraints (i.e. increasing pore dimensions), secondary reactions were identified, which reduce the selectivities to propene and butenes dramatically. These secondary reactions include mainly hydrogen transfer reactions to saturated hydrocarbons and cyclization/alkylation reactions to aromatics. By the selection of one-dimensional pore structures hydrogen transfer reactions and cyclization reactions were significantly reduced by suppression of the bulky transition states which are required for the formation of these by-products. Furthermore a strong influence of the reaction conditions on the formation of secondary reactions was observed for HZSM-5 with its three-dimensional pore structure. According to the experiments, the relative prevalence of the competing cracking mechanisms (monomolecular $v s$. bimolecular) strongly depends on the reaction conditions. High reaction temperatures and low ethene partial pressures favour monomolecular cracking and thus the formation of propene and butenes. Bimolecular cracking is preferred at lower reaction temperatures and higher ethene partial pressures, which leads to an increasing influence of secondary reactions with enhanced formation of saturated short chain hydrocarbons and aromatics.

\section{Acknowledgements}

Financial support for this work from NanoKat (Center for NanoStructured Catalysts) and the Center for Resource Efficient Chemistry and Feedstock Change (Supported by the Carl-ZeissFoundation) at the University of Kaiserslautern is gratefully acknowledged.

\section{References}

1 Y. Park, C. W. Lee, N. Y. Kang, W. C. Choi, S. Choi, S. H. Oh and D. S. Park, Catal. Surv. Asia, 2010, 14, 75-84.

2 J. S. Plotkin, Catal. Today, 2005, 106, 10-14.

3 A. Akah and M. Al-Ghrami, Appl. Petrochem. Res., 2015, 5, 377-392.

4 J. Mol, J. Mol. Catal. A: Chem., 2004, 213, 39-45.

5 J. C. Bricker, Top. Catal., 2012, 55, 19-20.

6 J. Q. Chen, A. Bozzano, B. Glover, T. Fuglerud and S. Kvisle, Catal. Today, 2005, 106, 103-107.

7 A. Behr, A. Kleyensteiber and U. Hartge, Chem. Ing. Tech., 2010, 82, 201-213.

8 T. Yamaguchi, Y. Tanaka and K. Tanabe, J. Catal., 1980, 65, 442-447.

9 M. Iwamoto, Molecules, 2011, 16, 7844-7863.

10 H. Oikawa, Y. Shibata, K. Inazu, Y. Iwase, K. Murai, S. Hyodo, G. Kobayashi and T. Baba, Appl. Catal., A, 2006, 312, 181-185.

11 B. Lin, Q. Zhang and Y. Wang, Ind. Eng. Chem. Res., 2009, 48, 10788-10795.

12 X. Ding, S. Geng, C. Li, C. Yang and G. Wang, J. Nat. Gas Chem., 2009, 18, 156-160.

13 T.-R. Koyama, Y. Hayashi, H. Horie, S. Kawauchi, A. Matsumoto, Y. Iwase, Y. Sakamoto, A. Miyyji, K. Motokura and T. Baba, Phys. Chem. Chem. Phys., 2010, 12, 2541-2554.

14 P. A. Jacobs and J. A. Martens, Studies in Surface Science and Catalysis, Elsevier, Amsterdam, 1987, vol. 33, pp. 19-20.

15 S. Ernst, J. Weitkamp, J. A. Martens and P. A. Jacobs, Appl. Catal., 1989, 48, 137-148.

16 S. Ernst, R. Kumar and J. Weitkamp, Am. Chem. Soc. Symp. Ser., 1989, 398, 560-573.

17 H. Robson and K. P. Lillerud, Verified Synthesis of Zeolitic Materials, Elsevier, Amsterdam, 2nd edn, 2011, pp. 115-116, and 147-148.

18 S. Brunauer, P. H. Emmett and E. Teller, J. Am. Chem. Soc., 1938, 60, 202-210.

19 M. M. J. Treacy and J. B. Higgins, Collection of Simulated XRD Powder Patterns for Zeolites, Elsevier, Amsterdam, 4th edn, 2001. 